Physics International 1 (1): 53-56, 2010

ISSN 1948-9803

(C) 2010 Science Publications

\title{
Michelson-Morley Experiment another Assessment
}

\author{
Koorosh Shahdaei \\ Klareborgsgatan 12 F, 41461 Gothenburg, Sweden
}

\begin{abstract}
Problem statement: Ehrenfest paradox may be the most basic phenomenon in relativity that has a long history marked by controversy, which still gets different interpretations by researches. This assessment is about another paradox in special relativity for observers in none inertial frame that perceiving Galilean transformation. Approach: This assessment reevaluates Michelson and Morley's famous experiment and Lorentz transformation by comparing different observers attached to different space-time frames. Results: The idea is to simulate a situation where an inertial frame will have comparable observation as a none-inertial frame. Hence it would be very close to an inertial frame at low velocity. Eventually it is shown that observation by none-inertial frame is Galilean transformation rather than Lorentz transformation. Conclusion: The outcome of physical experiments observed by inertial and none-inertial observers are completely different as they observe i.e., a fast-moving inertial frame which potentially contradicts Lorentz symmetry.
\end{abstract}

Key words: Special relativity paradox, Michelson-Morley

\section{INTRODUCTION}

Lorentz transformation and Special Relativity are based on the equivalence of all inertial reference frames and the invariance of the speed of light. The most general transformation of space and time coordinates can be derived using only the equivalence of all inertial reference frames and the symmetries of space and time.

The famous Michelson and Morley is about, interferometer that uses the wave property of interference to compare the time that light takes to travel along two paths perpendicular to each other. Since the original experiment, many similar experiments have been performed, but so far there is no evidence that speed of light would be invariant in vacuum regardless it is emitted form a stationary or moving body, for more contemporary experiments please refer to (Eisele et al., 2009).

Moreover Special Relativity, the entire theory is based on two postulates; firstly the laws of physics take the same form in all inertial frames, secondly in any inertial frame, the velocity of light $c$ in vacuum is the same whether the light is emitted by a body at rest or by a body in uniform motion, which furthermore concludes length contraction and time dilation.

This study is about proposing a new experiment that would reconstruct the observation of MichelsonMorley experiment to re-evaluate Galilean/Lorentz transformation in combination with the principal of special relativity.
To simplify, imagine a horse racing and a vehicle that moves parallel with the racing frame with same velocity, while broadcasting the racing event. As the speed is the same for racing frame and the vehicle what is observed in racing frame is equal in vehicle's frame. Imaging now an observer that is located in the center of the circuit and rotating with a speed aligned with center of the event. He/She will observer the racing as good as the vehicle frame as he/she is completely aligned with the racing frame in combination with rotational movement.

\section{MATERIAL AND METHODS}

Michelson Morley experiment: The Michelson Morley experiment is not consistent with Galilean/Newtonian physics. However its results are explained using Einstein's principle of relativity.

Following Galilean physics, consider that light travels at $\mathrm{c}$ with respect to a stationary medium e.g., Aether. Also suppose that paths $l_{1}$ and $l_{2}$ are equal and the entire spectrometer is stationary with respect to the medium that supports the wave motion of the light i.e., Aether. Let's consider a point in the interference pattern at which the phase difference is zero. This is the situation shown in Fig. 1 at left.

Now suppose that it move to the right at speed $\mathrm{v}$ with respect to so called Aether, then the paths are not equal any more refer to the Fig. 1, at right. 
Phy. Intl. 1 (1): 53-56, 2010

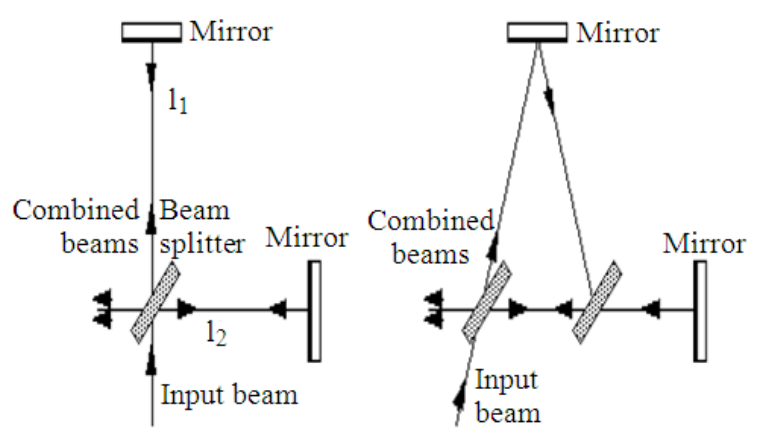

Fig. 1: Michelson Morley Experiment: interferometer of equal length arms $l_{1}$ and $l_{2}$ which are perpendicular, left-hand side rest frame and right-hand side moving frame, for any velocity $v$ of the moving frame it should be a fringe shift for the light in combined beams corresponding to $v$ due to unequal light paths

Furthermore it can be concluded that the transit times are no longer equal, so the time for the horizontal and near vertical directions are given by:

$\mathrm{t}_{2}=\frac{1}{\mathrm{c}+\mathrm{v}}+\frac{1}{\mathrm{c}-\mathrm{v}}=\frac{21}{\mathrm{c} \sqrt{1-\mathrm{v}^{2} / \mathrm{c}^{2}}}$

$\mathrm{t}_{1}=\frac{1}{\mathrm{c}^{2}-\mathrm{v}^{2}}+\frac{1}{\mathrm{c}^{2}-\mathrm{v}^{2}}=\frac{21}{\mathrm{c} \sqrt{1-\mathrm{v}^{2} / \mathrm{c}^{2}}}$

Lorentz transformation: Consider now Lorentz transformation, without considering too much technical details, let us consider two inertial reference frames $\mathrm{O}$ and $\mathrm{O}$ '. The reference frame $\mathrm{O}$ ' moves relative to $\mathrm{O}$ with velocity $\mathrm{v}$ along the $\mathrm{x}$ axis. Assume also that the coordinates $y$ and $\mathrm{z}$ perpendicular to the velocity are the same in both reference frames i.e., $\mathrm{y}=\mathrm{y}_{0}$ and $\mathrm{z}=\mathrm{z}_{0}$. So it is adequate to consider only transformation of the coordinates $\mathrm{x}$ and $\mathrm{t}$ from the reference frame $\mathrm{O}-\mathrm{x}^{\prime}=$ $f_{x}(x, t)$ and $t^{\prime}=f_{t}(x, t)$ in the reference frame O'. From translational symmetry of space and time, we conclude that the functions $\mathrm{f}_{\mathrm{x}}(\mathrm{x}, \mathrm{t})$ and $\mathrm{f}_{\mathrm{t}}(\mathrm{x}, \mathrm{t})$ must be linear functions.

Consequently the relative distances between two events in one reference frame must depend only on the relative distances in another frame:

$\mathrm{x}_{1}^{\prime}-\mathrm{x}_{2}^{\prime}=\mathrm{f}_{\mathrm{x}}\left(\mathrm{x}_{1}-\mathrm{x}_{2}, \mathrm{t}_{1}-\mathrm{t}_{2}\right)$

$t_{1}^{\prime}-t_{2}^{\prime}=f_{t}\left(x_{1}-x_{2}, t_{1}-t_{2}\right)$
One can conclude the standard Lorentz transformation will be in primed frame:

$x^{\prime}=\frac{x-v t}{\sqrt{1-v^{2} / c^{2}}}$

$\mathrm{t}^{\prime}=\frac{-\mathrm{xv} / \mathrm{c}^{2}+\mathrm{t}}{\sqrt{1-\mathrm{v}^{2} / \mathrm{c}^{2}}}$

Consider a combination of two consecutive Lorentz transformations with velocities $\mathrm{v}_{1}$ and $\mathrm{v}_{2}$, which represents the relativistic law of adding velocities, consequently one can conclude:

$v=\frac{v_{1}+v_{2}}{1+v_{1} v_{2} / c^{2}}$

If we consider:

$\mathrm{v} / \mathrm{c}=\beta$

and

$\left(1-v^{2} / c^{2}\right)-1 / 2=(1-\beta)^{-1 / 2}=\gamma$

Then for the primed frame the Lorentz contraction and time dilation would be equal to:

$\mathrm{L}^{\prime}=\mathrm{L} \gamma$

and

$\mathrm{t}^{\prime}=\mathrm{t} \gamma$

For detailed derivation of Lorentz transformation, please refer to (Katz 1964).

Experiment overview: In this experiment we will construct Michelson Morley experiment in such way that the experiment is observed by three different observers (Fig. 2). Observer $\mathrm{O}$ is stationary frame (inertial) attached to the laboratory that moved with velocity $\mathrm{v}_{\mathrm{x}}$ which performs the experiment. $\mathrm{O}^{\prime}$ is another inertial frame moving with same velocity (or less) and same direction as $\mathrm{O}$.

Observer O" has a circular motion compared to $\mathrm{O}$ and $\mathrm{O}^{\prime}$ and is viewing the experiment by a rotational movement synchronized and aligned with the center point of the experiment, additionally the center of its rotation is stationary compared to frame $\mathrm{O}$. Considering $\mathrm{r}$ is the distance of $\mathrm{O}$ " frame to experiment center point (i.e., beam splitter in Fig. 2), in addition $\mathrm{r}$ overlies $\mathrm{Y}^{\text {" }}$ 
axis. Also consider the line that crossing the beam splitter i.e., $l_{2}$ is parallel with $\mathrm{X}$ axis (Fig. 2). From observer O" point of view as the experiment proceeds; $l_{2}$ is rotating about X" axis with i.e., angle $\Phi$ (Fig. 2). Consequently; O" measures $r$ to vary along $\mathrm{Y}$ axis as $\Phi$ changes.

The observer O" will follow the experiment as it rotates in such way that its line of site to the experiment's center point is synchronized with the velocity $\left(\mathrm{v}_{\mathrm{x}}\right)$ of the experiment frame $\mathrm{O}$. If the distance of observer O" to the experiment is big enough then the circular displacement of the observer O" (telescope) will be very small and angle $\Phi$ will be close to $0^{\circ}$ and r will vary unnoticeably during the experiment and the results would be very similar to the inertial frame $\mathrm{O}^{\text {' }}$ that moves parallel with the experiment frame with same velocity, i.e., Galilean transformation.

\section{RESULTS}

Consider Michelson Morley (or other equivalent) experiment starts in left position of Fig. 2 and ends at the position in the right hand side, observer O" will experience that the laboratory in $\mathrm{O}$ frame tilts slightly i.e., clockwise as the experiment advances. But in this case there won't be any Lorentz contraction; as this experiment would very much simulate a Galilean transformation for observer O".

\section{DISCUSSION}

Considering Fig. 2, although observer O" sees the laboratory tilting slightly, but it is not the same as what happens in the right hand side of Fig. 1 i.e., length contraction in other word it is not a Lorentz transformation.

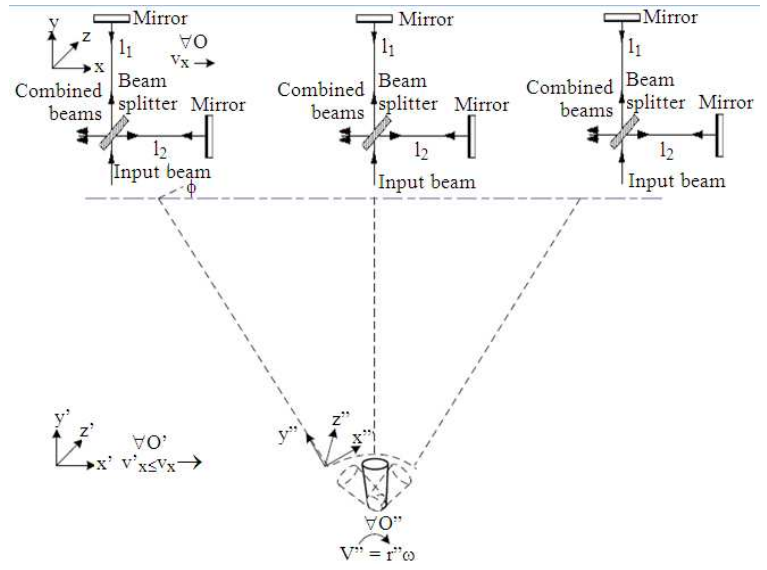

Fig. 2: Viewing three different positions of observers $\mathrm{O}$ and O"
Imagine the distance to telescope's image to be $\mathrm{r}$ " (Fig. 2), in a situation where the distance from O" frame to the laboratory is large, linear velocity of $\mathrm{O}$ " will be r" $\omega$ and for a small $r$ " and $\omega$ the linear velocity will be negligible compared to $v_{x}$ of the fast-moving $O$ frame. Consequently we can accept the approximation of fame O" to have a very low speed compared to $v_{x}$ of the $\mathrm{O}$ frame. As a result we can confirm that $\mathrm{O}$ " frame with low speed doesn't observe any length contraction which is contradictory to Lorentz contraction and special relativity.

As regards time dilation, if we would synchronize the clocks of O and O", by considering the time interval between light flashes of a moving clock fixed in frame $\mathrm{O}$ with its motion along the $\mathrm{x}$ axis, we only need observers along the $\mathrm{x}$ axis. In this case also both $\mathrm{O}$ and O" frames will have synchronized clocks as it would have happened in Galilean transformation.

If we consider the inertial frame O' (Fig. 2), to be at rest or with substantially lower speed than frame $\mathrm{O}$, then $\mathrm{O}$ ' frame will observe length contraction and time dilation, although O" with negligible linear speed compared to $\mathrm{O}$ frame; won't agree to length contraction and time dilation. This means if for instance a paircreation takes place at frame $\mathrm{O}$ the results will be contradictory for observers $\mathrm{O}^{\prime}$ and $\mathrm{O}^{\prime \prime}$ if special relativity principal is applied, i.e., if both $\mathrm{O}^{\prime}$ and $\mathrm{O}$ " have substantially low speed compared to frame $O$, special relativity principal won't hold the same for O' and $\mathrm{O}$ " frames. In other word $\mathrm{O}$ ' frame observers the wavelength of the two photons to have higher frequency and energy; but frame O" will observe same frequency and energy as $\mathrm{O}$ frame and consequently created pairs observed by $\mathrm{O}^{\prime}$ will put on kinetic energy as their masses are increased by factor $\gamma$ (Eq. 9), but frame O" will measure same wavelength for created pairs as frame $\mathrm{O}$ and as a result no kinetic energy. In other word these results are contradictory as two frames with low speed will observe different physical results, this means O' and O" frames will measure the energy of created pairs differently which is inconsistent with Special Relativity principal.

The question that the laws of physics should appear to be the same with regards to Lorentz symmetry (which is a fundament of special theory of relativity) will remain.

\section{CONCLUSION}

To sum up with this experiment, the general transformation of space and time coordinates and the symmetries of space and time can't be guaranteed. 
In conclusion we can agree that two frames with low speed will observe fast-moving frames differently for instance one can agree to Galilean transformation as the other one agrees to Lorentz transformation.

In mainstream physics, Lorentz symmetry in recent years embarked on to be questioned whether it is undeniably an exact symmetry of nature. The physicists are motivated primarily by the development of string and loop quantum gravity theories, which try to make gravity congenial with quantum physics but this allow for the possibility that Lorentz symmetry might not stick to precisely.

\section{REFERENCES}

Eisele, C., A.Y. Nevsky and S. Schiller, 2009. Laboratory test of the isotropy of light propagation at the 10_17 level. Phys. Rev. Lett., 103: 090401. DOI: 10.1103/PhysRevLett.103.090401

Katz, R., 1964. An Introduction to the Special Theory of Relativity. UNL, pp: 8-38. http://digitalcommons.unl.edu/physicskatz/49/ 